\title{
DESCARTE CONSCIENTE DE MEDICAMENTOS: RELATO DE EXPERIÊNCIA
}

\author{
Marina Guio Vassoleur ${ }^{1}$ \\ Daiana Freitas Ferreira ${ }^{2}$ \\ Raiane Facini Cesconetto ${ }^{3}$ \\ Wesley Careri Müller ${ }^{4}$ \\ Afrânio Aguiar Oliveira ${ }^{5}$ \\ Janaina Cecilia Oliveira Villanova ${ }^{6}$
}

Resumo: o presente trabalho teve como objetivo sensibilizar um grupo de alunos do ensino médio do município de Alegre (ES) sobre a importância do descarte consciente de medicamentos, com o intuito de torná-los multiplicadores das informações adquiridas. O projeto foi desenvolvido mediante a realização de aulas ministradas por alunos de graduação do curso de Farmácia da UFES e de um tutor da escola. Os alunos mostraram-se interessados e preocupados com a situação do descarte incorreto no município de Alegre, sendo estimulados a refletirem a partir de um problema mundial e a proporem alternativas para intervirem no descarte de medicamentos no âmbito local.

Palavras-chave: Uso racional de medicamentos; Descarte consciente; Educação ambiental.

\footnotetext{
1 Universidade Federal do Espírito Santo - UFES, Brasil. E-mail: marii-t1@hotmail.com.

2 Universidade Federal do Espírito Santo - UFES, Brasil. E-mail: daiafreitasferreira@hotmail.com.

3 Universidade Federal do Espírito Santo - UFES, Brasil. E-mail: raianefacini@hotmail.com.

4 Universidade Federal do Espírito Santo - UFES, Brasil. E-mail: wesley.muller@outloock.com.

5 Universidade Federal do Espírito Santo - UFES, Brasil. E-mail: afranioaguiar@bol.com.br.

${ }^{6}$ Universidade Federal do Espírito Santo - UFES, Brasil. E-mail: pharmacotecnica@yahoo.com.br.
} 